\title{
Preparation of porous carbon with zinc oxide template
}

\author{
HUO Pengwei ${ }^{1,}$, LIU Chongyang ${ }^{1, a}$ and MA Chunhong ${ }^{1, a}$
}

${ }^{1}$ School of chemistry \& chemical engineering, Jiangsu University, Zhenjiang China 210213

ahuopw1@163.com

\section{Keywords: zinc oxide; porous carbon; adsorption; ciprofloxacin}

Abstract. The porous carbon materials were prepared by the following processes of evaporation induction self-assembly (EISA), thermal polymerization process and calcination step at high temperature with the nano-zinc oxide as a hard template and phenol resin as carbon source. The as-prepared porous carbon materials were characterized by scanning electron microscopy (SEM), transmission electron microscopy (TEM), $\mathrm{N}_{2}$ adsorption/desorption isotherms (BET) and Fourier transform infrared spectroscopy (FT-IR). The adsorption performance was investigated via removal ciprofloxacin antibiotic. The results showed that the more the amount of zinc oxide template was added in the prepared process of porous carbon, the clearer pore channel of as-prepared porous carbon was exhibited. When the dosage of zinc oxide reach $0.4 \mathrm{~g}$, it is well formed a preferably porous carbon, the higher adsorption rate of ciprofloxacin with $100 \mathrm{~mL}$ of $15 \mathrm{mg} / \mathrm{L}$ could reach $71.2 \%$.

\section{Introduction}

Adsorption materials were considerable attentions and have been large reported. A variety of novel morphologies such as clay mineral, activated sludge, the soil, molecular printed polymer, carbon materials $[1,2]$, et al., they are also presented excellent adsorption performances. Among these materials, carbon materials are widely investigated due to the rich source and easy to prepare. Especially, the porous carbon materials are generally fabricated using porous silica as hard template via synthetic method of nano-perfusion. Porous channel of porous silica is perfused with carbon source. Silica/carbon composites were obtained after carbonization at high temperature. Porous carbon was obtained after removing silicon oxide with hydrofluoric acid or sodium hydroxide solution [3, 4]. Jiang et al. showed that hierarchical porous carbons was synthesized via nano-casting method, using hollow mesoporous silica spheres as hard template and phenolic resin as carbon source [5]. Although the hard template could effectively synthesize the porous carbon, the silica must remove by the hydrofluoric acid or concentrated alkaline which maybe pollute or threaten the environment. However, the other oxides, such as $\mathrm{ZnO}$ and $\mathrm{Al}_{2} \mathrm{O}_{3}$ which could be used as the hard template materials, are easy to remove by the diluted hydrochloric acid. Especially, the $\mathrm{ZnO}$ which possesses chemical stability and non-toxicity feature is better to prepare the nano-structure and obtain the unique morphology, and has exhibited great application potential in solar cell, amplifier, sensor, and so on[6-10].

In this work, we report that porous carbon was synthesized by using zinc oxide as hard template and phenolic resin as carbon source, through evaporation induced self-assembly (EISA), thermal fusion process and high temperature calcination. At last, the hard template was easy to remove by the diluted hydrochloric acid, and the porous carbon was obtained.

\section{Experimental}

Nano-sized ZnO synthesis. Citric acid, zinc nitrate and ethylene glycol were dissolved in deionized water with mole ratio $3: 1: 12$. The mixed solution was stirred for $2 \mathrm{~h}$, after that the mixed solution was put in the drying oven and maintained at $80{ }^{\circ} \mathrm{C}$ for $12 \mathrm{~h}$. Then, the temperature was adjusted to $130{ }^{\circ} \mathrm{C}$ and kept for $7 \mathrm{~h}$. Last, the samples were calcined at $900^{\circ} \mathrm{C}$ for $4 \mathrm{~h}$ and naturally cooled to room temperature, and the nano-sized $\mathrm{ZnO}$ was obtained. 
Porous carbon synthesis.Phenolic resin and F127 of mole ratio 1:1 were fully dissolved in absolute ethyl alcohol and kept stirring for $3 \mathrm{~h}$ to dissolve fully under the water bath condition of at $50^{\circ} \mathrm{C}$. A certain amount of nano-sized $\mathrm{ZnO}$ was uniformly distributed in the above solution, and remained magnetic stirring for 30 min under the water bath condition of at $50^{\circ} \mathrm{C}$. Then, the mixed solution was put in the Petri dish and fully evaporated at room temperature. The samples were solidified at $100^{\circ} \mathrm{C}$ for $24 \mathrm{~h}$ in drying oven, and it was still sequentially solidified for $12 \mathrm{~h}$ at $150{ }^{\circ} \mathrm{C}$. The rough samples were obtained and pulverized uniformly, and the samples were calcined in tube furnace without oxygen at $900^{\circ} \mathrm{C}$. At last, the above samples were put into $1 \mathrm{~mol} / \mathrm{L}$ hydrochloric acid solution and stirred $12 \mathrm{~h}$, filtration and drying in the oven at $80^{\circ} \mathrm{C}$. The porous carbon was obtained.

Structure and textual characterization. The morphology observation of samples was examined with JSM-6480 scanning electron microscopy (SEM, JEOL Ltd., Japan). TEM image was collected through JEM-2100 electron microscope set accelerating voltage of $200 \mathrm{Kv}$. Specific surface area, pore volume and pore size distribution was measured by specific surface area analyzer and gap Tnstar 113020 (Mackcompany, American). Fourier transform infrared (FT-IR) spectra were recorded on a Nicolet Nexus 470 FT-IR (America thermo-electricity Company) with $2 \mathrm{~cm}^{-1}$ resolution in the range $400-4000 \mathrm{~cm}^{-1}$, using $\mathrm{KBr}$ pellets.

Determination of porous carbon adsorption. A certain amount of adsorbent material was put into $100 \mathrm{~mL} 15 \mathrm{mg} / \mathrm{L}$ ciprofloxacin solution at room temperature. The sampling analysis was conducted in $10 \mathrm{~min}$ interval. The adsorption rate (AR) was calculated by the following formula (1):

$$
\mathrm{AR}=\left(1-\mathrm{A}_{\mathrm{i}} / \mathrm{A}_{0}\right) \times 100 \% \text {. }
$$

Where $A_{0}$ is the initial absorbency of ciprofloxacin antibiotic solution and $A_{i}$ is the absorbance of reaction solution.

\section{Results and discussions}

SEM and TEM. The morphology and micro-structure of zinc oxide and porous carbon materials were characterized by SEM and TEM. The results showed in Figure 1, it can be clearly seen that the carbon without zinc oxide was disordered and the surface was rough, but it possessed few small holes. Compared with Figure 1-a, it can be easily observed that the surface of porous carbon possess a few holes (see Figure 1-b) and the specific surface area was also correspondingly enlarged. But in the Figure 1-c, it can be seen that the surface of the samples increased a lot of apertures with adding large dosage of zinc oxide. This was may be due to that zinc oxide and carbon were closed together in the process of calcination in the tube furnace. The surface of porous carbon was correspondingly generated a great deal of small holes after removing the template of zinc oxide using hydrochloric acid.

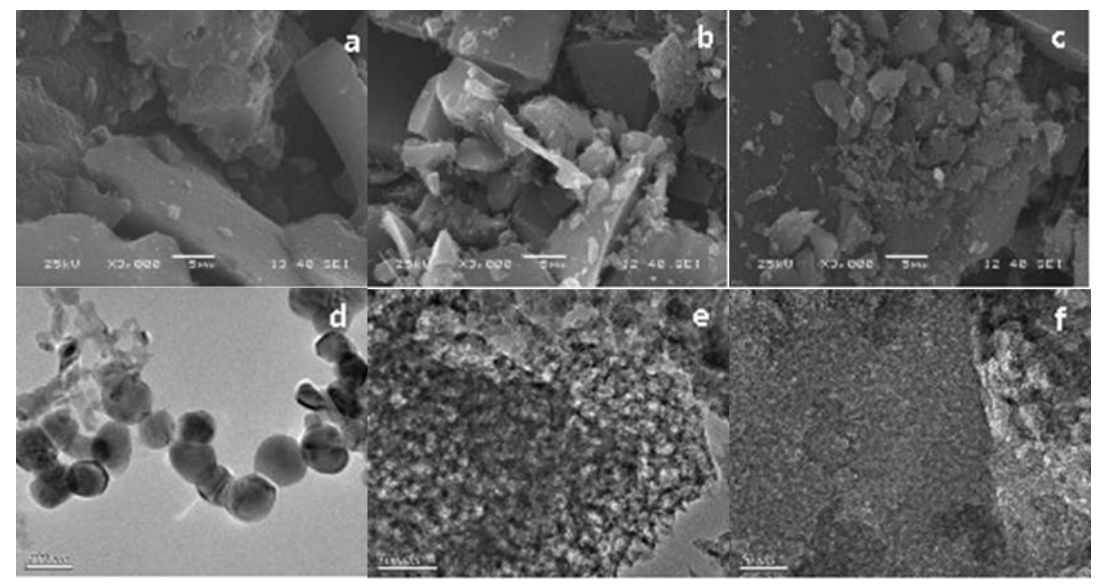

Figure.1 SEM images of samples ( $a$. porous carbon without zinc oxide; b. porous carbon of adding $0.1 \mathrm{~g}$ zinc oxide; c. porous carbon of adding $0.4 \mathrm{~g}$ zinc oxide; TEM images of samples (d. zinc

oxide; e. porous carbon of adding $0.4 \mathrm{~g}$ zinc oxide; $\mathrm{f}$. porous carbon of without zinc oxide )

The TEM images of Figure 1-d, e and f were showed the zinc oxide, porous carbon of adding $0.4 \mathrm{~g}$ zinc oxide and porous carbon without adding zinc oxide, respectively. It can be clearly seen that the morphology of zinc oxide was exhibited uniformly spherical structure (Figure 1-d). This is may 
be due to that zinc nitrate and citric acid were scattered uniformly in ethylene glycol and appropriate calcined temperature will be better for formed the spherical structure. Compared with Figure 1-f, surface of porous carbon possessed many irregular small holes approximately diameter of $50 \mathrm{~nm}$ in the Figure 1-e. The mainly reason due to the template of zinc oxide was removed, many large holes appeared on the surface of carbon. There were few small holes appeared in the Figure1-f. The reason may be due to that there was no zinc oxide in the prepared process, and there was only F127 in the composite samples.

FT-IR. Figure 2 showed the FT-IR spectra of porous carbon, carbon and zinc oxide. Compared to the curve of $\mathrm{ZnO}$, the peaks of $\sim 3400 \mathrm{~cm}^{-1}$ were assigned to relatively weak vibration peak of -OH, which showed that surface of materials contained -OH group in the curves of porous carbon and carbon. The peak between $2000 \mathrm{~cm}^{-1}$ and $2500 \mathrm{~cm}^{-1}$ belonged to $\mathrm{C}=\mathrm{C}$ stretching vibration caused by substitute benzene ring of phenolic resin. Peaks between $2700 \mathrm{~cm}^{-1}$ and $3000 \mathrm{~cm}^{-1}$ belonged to stretching vibration of $\mathrm{C}-\mathrm{H}$ which came from $\mathrm{F} 127,1100 \mathrm{~cm}^{-1}$ peak attributed to $\mathrm{C}-\mathrm{O}$ stretching vibration peak of F127. The peaks near $1500 \mathrm{~cm}-1$ were vibration peak of $\mathrm{C}-\mathrm{C}$ bond.
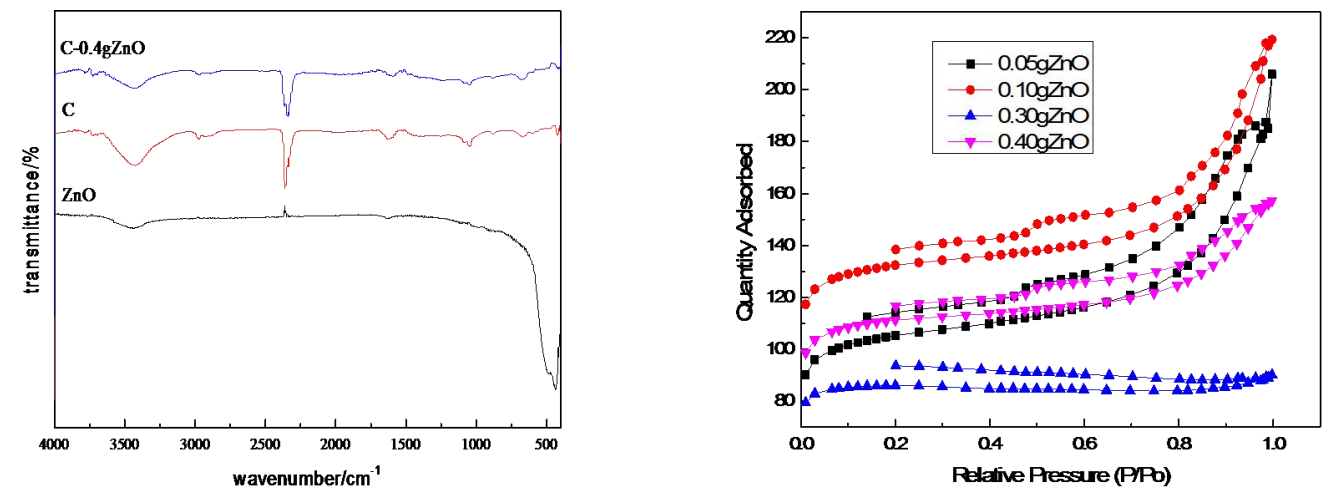

Figure 2 FT-IR spectra of as-prepared samples Figure $3 \mathrm{~N}_{2}$ adsorption/desorption isotherms samples BET. Figure 3 shows $\mathrm{N}_{2}$ adsorption/desorption isotherms of the obtained porous carbon with different dosage of $\mathrm{ZnO}$. In the Figure 3, there were certain hysteresis phenomena with hysteresis loop in all these curves, which indicated that cylindrical narrow channel with uniform size and regular shape had been formed. Bumps that capillary condensation phenomenon caused in the scope of relative pressure, exhibited that the four samples had relatively big pore size distribution due to relative large specific surface area formed in the fabricating process. Compared with four isotherms, it was found that the more added zinc oxide, the more obvious formed bump phenomenon, indicating the more added zinc oxide, which leaded to form more pore passage and larger specific surface area after removing zinc oxide.

Adsorption performance. Adsorbent performance of porous carbon, carbon and zinc oxide for ciprofloxacin were investigated. The results were illustrated in Figure 4. From Figure 4, it can be clearly found that the porous carbon possessed the highest adsorption rate. The reason was mainly depended on the porous structure which prepared with adding the nano-sized zinc oxide template possessed the larger specific surface area and pore channel. Therefore, it possessed higher adsorption performance than of carbon and spherical zinc oxide. 


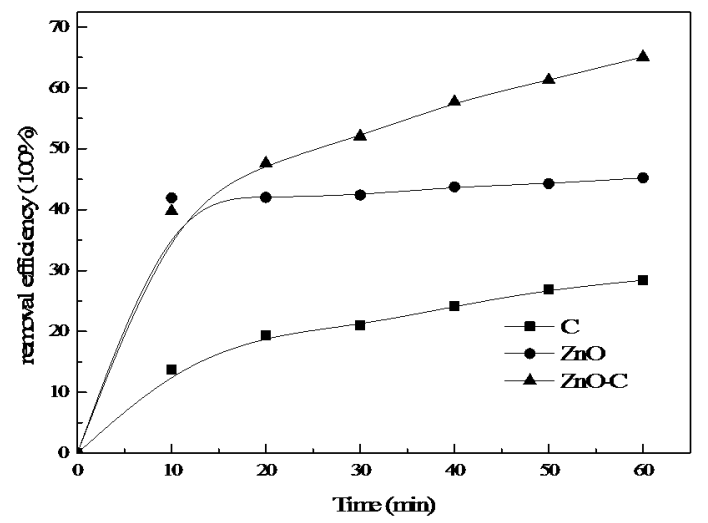

Figure 4 Adsorption performance of carbon, porous carbon and zinc oxide

Figure 5 showed the influences on adsorption of ciprofloxacin with different dosage of zinc oxide for prepared porous carbon. From Figure 5, it can be obviously found that the adsorption rate of ciprofloxacin with porous carbon adding $0.4 \mathrm{~g}$ zinc oxide was highest and the worst one was the porous carbon with adding $0.05 \mathrm{~g}$ zinc oxide. Indicating that the more added dosage of zinc oxide, the better adsorption rate of ciprofloxacin. The reason mainly due to the appropriate dosage of zinc oxide added into the prepared processes, the porous carbon could form the better porous structure, it could obtain the large specific surface area, which increased the adsorption rate of ciprofloxacin.

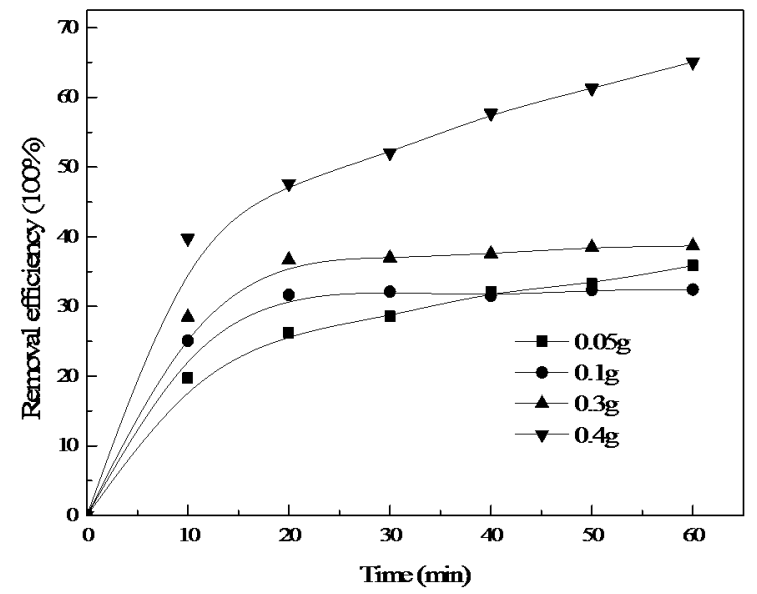

Figure 5 The influences of different dosage of zinc oxide on porous carbon adsorption

Different dosage of porous carbon on adsorption of ciprofloxacin was investigated. The result was demonstrated in Figure 6. From Figure 6, it can be clearly seen that the adsorption rate of ciprofloxacin with $0.2 \mathrm{~g}$ porous carbon was the best, and the $0.05 \mathrm{~g}$ porous carbon showed the lowest for adsorption rate of ciprofloxacin. Indicating that the more dosage of porous carbon was added, the higher adsorption rate of ciprofloxacin. When the porous carbon dosage reached $0.2 \mathrm{~g}$, the adsorption rate almost reached 100\% in 30 minutes. But increasing the adsorption reaction time on ciprofloxacin, the adsorption rate for ciprofloxacin could not be significantly increased. 


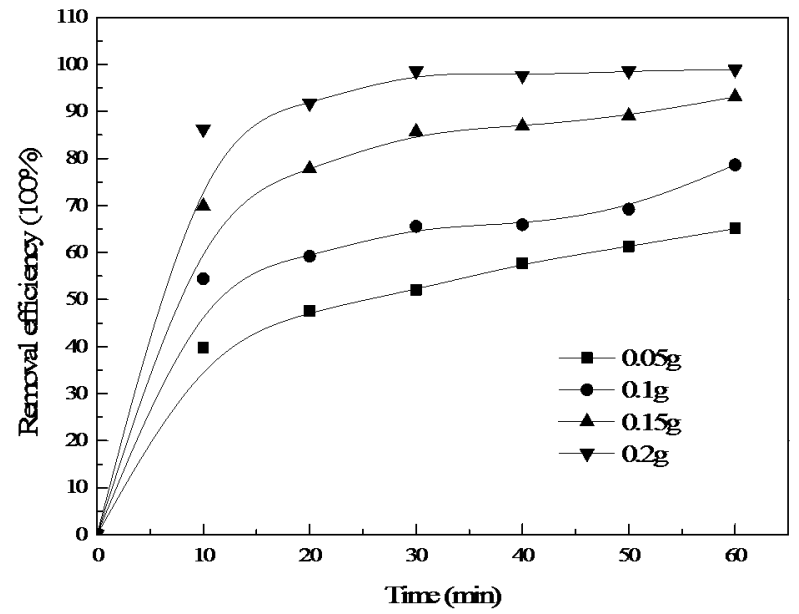

Figure 6 The adsorption influence of different dosage of porous carbon

\section{Conclusions}

This work was mainly showed the preparation and adsorption on ciprofloxacin of porous carbon. The zinc oxide was chosen as hard template to replace the traditional silica template. And the ciprofloxacin was selected as pollutants. Results indicated the more added zinc oxide, the larger obtained pore and specific surface area of porous carbon, which caused to increase adsorption rate. When added $0.4 \mathrm{~g}$ zinc oxide, the adsorption rate could reach $75.28 \%$. The more porous carbon was used, the better adsorption performance on ciprofloxacin was. When the added dosage porous carbon of $0.2 \mathrm{~g}$, the adsorption rate of ciprofloxacin almost reached $100 \%$.

\section{Acknowledgements}

This work was financially supported by financial support of the Natural Science Foundation of Jiangsu Province (BK20130489, BK20151349).

\section{References}

[1] O. A.Conchi, G. P. Joaquina and J. B.Teresa: Adsorption Vol.11(2011),p.421

[2] M. V. Ilina, A. V. Timofeeva, V. T. Ivanova, E. I. Burtseva, L. A. BaratovaI. Yu. Sapurina and G. S. Katrukha: Applied Biochemistry and Microbiology Vol.48(2012),p.699

[3] S.Jun, S. H. Joo and R.Ryoo:Journal of the American Chemical Society Vol.122(2000),p.10712

[4] F. S.Cristiam, J.Maguy, L. G.Jean and S.Ligia:Microporous and Mesoporous Materials Vol.173(2013),p.53

[5] L.X.Jiang, Q.L.Cheng, Y.M.Xia, V.Pavlinek, P.Saha and C.Z.Li: Journal of Materials Science Vol.49(2014),p.7489

[6] J.Wu, J.Xia, J.Chen, W.Lei, B.P.Wang, Y. F. Zhang and D. D.Engelsena: Nanoscience Nanotechnology Letters Vol.6(2014),p.1005

[7] J. J.Lee, G. Z.Xing, J. B.Yi, T.Chen, M.Ionescu and S.Li: Applied Physics Letters Vol.104(2014),p.012405

[8] G. Z.Xing, D. D.Wang, C.J.Cheng, M.He, S. Li and T.Wu: Applied Physics Letters Vol.103(2013),p. 022402

[9] R.Kumar, G.Kumar and A.Umar: Nanoscience Nanotechnology Letters Vol.6(2014),p.631

[10] Y.L.Lai, M.Meng,Y.F.Yu, X.T.Wang and T.Ding: Applied Catalysis B: Environmental Vol.105(2011),p.335 